\title{
The Average Tree Solution for Cycle-free Graph Games
}

Citation for published version (APA):

Herings, P. J. J., van der Laan, G., \& Talman, A. J. J. (2008). The Average Tree Solution for Cycle-free Graph Games. Games and Economic Behavior, 62, 77-92. https://doi.org/10.1016/j.geb.2007.03.007

Document status and date:

Published: 01/01/2008

DOI:

10.1016/j.geb.2007.03.007

Document Version:

Publisher's PDF, also known as Version of record

Document license:

Taverne

Please check the document version of this publication:

- A submitted manuscript is the version of the article upon submission and before peer-review. There can be important differences between the submitted version and the official published version of record.

People interested in the research are advised to contact the author for the final version of the publication, or visit the DOI to the publisher's website.

- The final author version and the galley proof are versions of the publication after peer review.

- The final published version features the final layout of the paper including the volume, issue and page numbers.

Link to publication

\footnotetext{
General rights rights.

- You may freely distribute the URL identifying the publication in the public portal. please follow below link for the End User Agreement:

www.umlib.nl/taverne-license

Take down policy

If you believe that this document breaches copyright please contact us at:

repository@maastrichtuniversity.nl

providing details and we will investigate your claim.
}

Copyright and moral rights for the publications made accessible in the public portal are retained by the authors and/or other copyright owners and it is a condition of accessing publications that users recognise and abide by the legal requirements associated with these

- Users may download and print one copy of any publication from the public portal for the purpose of private study or research.

- You may not further distribute the material or use it for any profit-making activity or commercial gain

If the publication is distributed under the terms of Article $25 \mathrm{fa}$ of the Dutch Copyright Act, indicated by the "Taverne" license above, 


\title{
The average tree solution for cycle-free graph games
}

\author{
P. Jean Jacques Herings ${ }^{\mathrm{a}, *}$, Gerard van der Laan ${ }^{\mathrm{b}}$, Dolf Talman ${ }^{\mathrm{c}}$ \\ a Department of Economics, Maastricht University, P.O. Box 616, 6200 MD Maastricht, The Netherlands \\ ${ }^{\mathrm{b}}$ Department of Econometrics and Tinbergen Institute, Vrije Universiteit, De Boelelaan 1105, \\ 1081 HV Amsterdam, The Netherlands \\ ${ }^{\mathrm{c}}$ Department of Econometrics \& Operations Research and CentER, Tilburg University, P.O. Box 90153, \\ 5000 LE Tilburg, The Netherlands
}

Received 1 September 2006

Available online 23 April 2007

\begin{abstract}
In this paper we study cooperative games with limited cooperation possibilities, represented by an undirected cycle-free communication graph. Players in the game can cooperate if and only if they are connected in the graph. We introduce a new single-valued solution concept, the average tree solution. Our solution is characterized by component efficiency and component fairness. The interpretation of component fairness is that deleting a link between two players yields for both resulting components the same average change in payoff, where the average is taken over the players in the component. The average tree solution is always in the core of the restricted game and can be easily computed as the average of $n$ specific marginal vectors, where $n$ is the number of players. We also show that the average tree solution can be generated by a specific distribution of the Harsanyi dividends.
\end{abstract}

(C) 2007 Elsevier Inc. All rights reserved.

JEL classification: $\mathrm{C} 71$

Keywords: TU-game; Communication structure; Myerson value; Fairness; Marginal vector

\footnotetext{
* Corresponding author. Fax: +31433884878.

E-mail addresses: p.herings@ @algec.unimaas.nl (P.J.J. Herings), glaan@feweb.vu.nl (G. van der Laan), talman@uvt.nl (D. Talman).

URL: http://www.fdewb.unimaas.nl/algec/.
} 


\section{Introduction}

A situation in which a finite set of players can obtain certain payoffs by cooperation can be described by a cooperative game with transferable utility, or simply a TU-game, being a pair consisting of a finite set of players and a characteristic function on the set of coalitions of players that assigns a payoff to each coalition of players. This payoff is the total payoff the coalition can obtain if they agree to cooperate. A payoff vector indicates a distribution of payoffs to the players in a game. A solution for TU-games is a mapping that assigns to every game a set of payoff vectors. A classical solution is the core, see Gillies (1953), which assigns to any game the set of undominated efficient payoff vectors. The best known single-valued solution is the Shapley value, see Shapley (1953), assigning to any game the average of all marginal vectors.

In this paper we study TU-games with limited cooperation possibilities, represented by an undirected communication graph as introduced by Myerson (1977). The vertices in the graph represent the players and the edges represent the communication links between the players. Players can only cooperate if they are connected. This yields a so-called graph game, given by a triple consisting of a finite set of players, a characteristic function on the set of coalitions of players, and a set of edges (communication links) between the players. Since in graph games only connected coalitions can cooperate, the core reduces to the set of component efficient payoff vectors that are not dominated by any connected coalition, and equals the core of the so-called restricted game as defined by Myerson (1977).

A solution for graph games is a mapping that assigns to every graph game a set of payoff vectors. The best-known single-valued solution for graph games is the Myerson value, a solution that assigns to any graph game the Shapley value of the restricted game. The Myerson value can be characterized by component efficiency and fairness. The latter property says that deleting a link between two players yields for both players the same change in payoff. Clearly, the Myerson value is the average of all marginal vectors of the reduced game and is guaranteed to be in the core if the restricted game is convex. Alternative characterizations of the Myerson value have been given in Myerson (1980) and Borm et al. (1992). In the latter paper also another solution for graph games has been proposed, the so-called positional value, see also Meessen (1988). This value is characterized by component efficiency and by balanced total threats, see Slikker (2005). The balanced total threat property says that the total threat of any player towards another player equals the total threat of that player towards the first player, where the total threat of a player towards another player is the sum over all links of the first player of payoff differences the second player experiences if such a link is broken.

We keep component efficiency as an axiom and replace Myerson's fairness by an alternative fairness property, to be called component fairness. This property says that deleting a link between two players yields for both resulting components the same average change in payoff, where the average is taken over the players in the component. Component fairness therefore emphasizes that in a cooperative graph game the losses associated to severing a link should be attributed to the two components, rather than to the two individual players whose link is deleted, and that the losses should be proportional to the size of the component. We show that component efficiency and component fairness characterize a new single-valued solution concept for cycle-free graph games, to be called the average tree solution.

It is easy to calculate the average tree solution. From Kaneko and Wooders (1982) and Le Breton et al. (1992) it follows that the core of the restricted game is not empty if the graph contains no cycles (a cycle-free graph) and the characteristic function is superadditive, see also Demange (1994). For an arbitrarily taken node in the graph, a cycle-free graph induces a tree with 
the given node as root. Demange assigns to any node taken as root a particular marginal vector and shows that this vector is in the core of the restricted game. Interpreting the tree as a hierarchy on the set of players, Demange (2004) argues that hierarchies yield stability, in the sense that there exist undominated payoff vectors under the mild condition of superadditivity, providing a rationale for the fact that a group organizes itself in a hierarchy to achieve coordination.

We will show that the average tree solution equals the average of the marginal vectors, for each player one, considered by Demange. From this it follows that the average tree solution belongs to the core of the (restricted) game if the game itself is superadditive. So, contrary to the Myerson value and the position value, in case of superadditivity the average tree solution is always an element of the core.

As a final way of calculating the average tree solution, we show that the average tree solution can be obtained from a particular distribution of the Harsanyi dividends corresponding to connected coalitions, see Harsanyi (1959). For the Myerson value dividends are allocated uniformly, and for the position value proportional to the degree of players. For the average tree solution, the dividends are allocated proportional to the number of players represented by a player. Each player represents himself and the players outside the coalition that are connected to him in the communication graph.

This paper has been organized as follows. In Section 2 we give some preliminary notions of graph theory. In Section 3 we prove that component efficiency and component fairness uniquely determine a solution on the class of cycle-free graph games. We also show that it equals the average of $n$ specific marginal vectors, where $n$ is the number of players. In Section 4 we show that in case the game is superadditive the average tree solution lies in the core, which implies that the average tree solution is the unique solution that is characterized by component fairness and stability. Section 5 discusses the average tree solution in terms of the distribution of Harsanyi dividends. Section 6 concludes.

\section{Preliminaries}

A cooperative game with transferable utility, or simply a TU-game, is a pair $(N, v)$, where $N$ is a finite set of players and $v: 2^{N} \rightarrow \mathbb{R}$ is a characteristic function on $N$ such that $v(\emptyset)=0$. For any subset $S \in 2^{N}, v(S)$ is the worth of coalition $S$. We assume that $N$ is a set of $n$ players, indexed by $i=1, \ldots, n$, and we denote a game $(N, v)$ shortly by its characteristic function $v$.

A payoff vector $x \in \mathbb{R}^{n}$ of a game $v$ is an $n$-dimensional vector giving a payoff $x_{i} \in \mathbb{R}$ to any player $i \in N$. In the following we denote $x(S)=\sum_{i \in S} x_{i}, S \in 2^{N}$. A payoff vector $x$ is efficient if it exactly distributes the worth $v(N)$ of the 'grand coalition' $N$, i.e. if $x(N)=v(N)$. A solution for TU-games is a mapping $F$ that assigns to every TU-game $v$ a set of payoff vectors $F(v) \subseteq \mathbb{R}^{n}$. A solution $F$ is efficient if for any game $v$ every element in $F(v)$ is efficient. The core is the most well-known efficient solution concept. For a TU-game $v$ the core is defined by

$$
C(v)=\left\{x \in \mathbb{R}^{n} \mid x(N)=v(N), x(S) \geqslant v(S), S \in 2^{N}\right\} .
$$

For some permutation $\pi$ on $N$, the corresponding marginal vector $m^{\pi}(v) \in \mathbb{R}^{n}$ assigns to every player $i$ a payoff $m_{i}^{\pi}(v)=v\left(\pi^{i} \cup\{i\}\right)-v\left(\pi^{i}\right)$, where $\pi^{i}=\{j \in N \mid \pi(j)<\pi(i)\}$, i.e. $\pi^{i}$ is the set of players preceding $i$ in the permutation $\pi$. The best known single-valued solution is the Shapley value, which assigns to any game $v$ the average $\psi(v)$ of all marginal vectors. Since every marginal vector is efficient, the Shapley value is an efficient solution.

A game $v$ is convex if $v(S \cup T)+v(S \cap T) \geqslant v(S)+v(T)$ for all $S, T \in 2^{N}$ and superadditive if $v(S \cup T) \geqslant v(S)+v(T)$ for all $S, T \in 2^{N}$ such that $S \cap T=\emptyset$. The core of a game is equal to 
the convex hull of all marginal vectors if and only if the game is convex. In that case the Shapley value is an element of the core. In general, the Shapley value may be outside the core, even if the core is non-empty. The core is non-empty if and only if the game is balanced, see Bondareva (1963).

In this paper we study cooperative games with limited cooperation possibilities, represented by an undirected communication graph as introduced by Myerson (1977). An undirected graph is a pair $(N, L)$ where $N$ is a set of nodes ${ }^{1}$ and $L$ is a collection of edges, i.e. $L \subseteq\{\{i, j\} \mid$ $i, j \in N, i \neq j\}$ is a collection of subsets of $N$ such that each element of $L$ contains precisely two distinct elements of $N$. Node $j$ is adjacent to node $i$ if $\{i, j\} \in L$. Because the elements of $L$ represent binary communication links between the players, in the sequel we call them links instead of edges.

For $K \in 2^{N}$, the graph $(K, L(K))$ with $L(K)=L \cap(K \times K)$ is called the subgraph of $(N, L)$ on $K$. The number of nodes in $K$ being adjacent to node $i \in K$ in subgraph $(K, L(K))$ is called $i$ 's degree in $(K, L(K))$ and is given by $d_{K}^{L}(i)=|\{j \in K \mid\{i, j\} \in L(K)\}|$.

A sequence of $k$ different nodes $\left(i_{1}, \ldots, i_{k}\right)$ is a path in a graph $(N, L)$ if $\left\{i_{h}, i_{h+1}\right\} \in L$ for $h=1, \ldots, k-1$. Two nodes $i, j \in N$ are connected in the graph $(N, L)$ if $i=j$ or there exists a path $\left(i_{1}, \ldots, i_{k}\right)$ with $i_{1}=i$ and $i_{k}=j$. A graph $(N, L)$ is connected if any two nodes $i, j \in N$ are connected in $(N, L)$. In a given graph $(N, L)$, a set of nodes $K$ is said to be a connected subset of $N$ when the subgraph $(K, L(K))$ is connected. A subset $K$ of $N$ is a component of $(N, L)$ if the subgraph $(K, L(K))$ is maximally connected, i.e. $(K, L(K))$ is connected and for any $j \in N \backslash K$, the subgraph $(K \cup\{j\}, L(K \cup\{j\}))$ is not connected. Note that the collection of components of $(N, L)$ forms a partition of $N$. The collection of all connected subsets of $K$ in the subgraph $(K, L(K))$ of $(N, L)$ is denoted by $C^{L}(K)$. The collection of all components of $(K, L(K))$ is denoted by $C_{m}^{L}(K)$. Notice that $C_{m}^{L}(K) \subseteq C^{L}(K)$.

A sequence of nodes $\left(i_{1}, \ldots, i_{k+1}\right)$ is a cycle in $(N, L)$ if

(i) $k \geqslant 2$,

(ii) all nodes $i_{1}, \ldots, i_{k}$ are different elements of $N$,

(iii) $i_{k+1}=i_{1}$, and

(iv) $\left\{i_{h}, i_{h+1}\right\} \in L$ for $h=1, \ldots, k$. A graph $(N, L)$ is cycle-free when it does not contain any cycle.

The combination of a TU-game and a communication graph results in a so-called graph game, given by a triple $(N, v, L)$ with $N$ the set of players, $v$ the characteristic function and $L$ the set of edges (communication links) in the graph $(N, L)$ on the set of nodes $N$. As for TU-games, we often omit $N$ in our notation. In a graph game only the connected coalitions are able to cooperate. For a graph game $(v, L)$, a payoff vector $x \in \mathbb{R}^{n}$ is component efficient if it holds that $\sum_{i \in K} x_{i}=v(K)$ for each $K \in C_{m}^{L}(N)$. The core $C(v, L)$ of a graph game $(v, L)$ reduces to the set of component efficient payoff vectors that are not dominated by any connected coalition,

$$
C(v, L)=\left\{x \in \mathbb{R}^{n} \mid x(S)=v(S), S \in C_{m}^{L}(N), \text { and } x(S) \geqslant v(S), S \in C^{L}(N)\right\} .
$$

\footnotetext{
1 Since in this paper the nodes in a graph represent the players in a game we use the same notation for both the set of nodes and the set of players.
} 
The core $C(v, L)$ of the graph game $(v, L)$ equals the core $C\left(v^{L}\right)$ of the so-called restricted game $v^{L}$, defined by Myerson (1977) as

$$
v^{L}(S)=\sum_{T \in C_{m}^{L}(S)} v(T), \quad S \in 2^{N} .
$$

\section{Component fairness}

In this section we introduce the axiom of component fairness condition and prove that there is a unique solution on the class of cycle-free graph games that satisfies the axioms of component efficiency and component fairness.

A single-valued solution for cycle-free graph games is a function $f$ that assigns to every cycle-free graph game $(v, L)$ a unique payoff vector $f(v, L) \in \mathbb{R}^{n}$.

The Myerson value $\mu$ is the solution that assigns to any graph game the Shapley value of the restricted game, so $\mu(v, L)=\psi\left(v^{L}\right)$. Clearly, the Myerson value is the average of all marginal vectors of $v^{L}$ and is guaranteed to be in the core if the restricted game is convex.

The Myerson value can be characterized by component efficiency and fairness. First we recall the axiom of component efficiency as introduced in Myerson (1977).

Axiom 3.1 (Component efficiency). A solution $f$ on the class of cycle-free graph games is component efficient if for any graph game $(v, L)$ it holds that $\sum_{i \in K} f_{i}(v, L)=v(K)$ for each $K \in C_{m}^{L}(N)$.

Component efficiency requires that a solution assigns to any component $K$ the payoff $v(K)$.

Axiom 3.2 (Fairness). A solution $f$ on the class of cycle-free graph games satisfies fairness if, for any $(v, L)$, for any link $\{i, j\} \in L$, it holds that

$$
f_{i}(v, L)-f_{i}(v, L \backslash\{i, j\})=f_{j}(v, L)-f_{j}(v, L \backslash\{i, j\}) .
$$

The latter property says that deleting a link between two players yields for both players the same change in payoff.

For a component $K$ of a graph $(N, L)$ and a link $\{i, j\} \in L(K)$, let $K^{h}$ be the component in ( $N, L \backslash\{i, j\}$ ) containing $h$, where $h=i, j$. Clearly, $K^{h}$ is the subset of $K$ of players connected to $h$ in the graph that results after deleting the link $\{i, j\}$. The Myerson fairness condition requires that $f_{i}(v, L)-f_{i}(v, L \backslash\{i, j\})=f_{j}(v, L)-f_{j}(v, L \backslash\{i, j\})$ for any $\{i, j\} \in L$ and thus states that two players $i$ and $j$ linked directly together face the same loss in payoff when the link between them is deleted. In other words, in the graph $(N, L \backslash\{i, j\})$ the players in $K^{i}$ cannot interact with the players in $K^{j}$, but when the players in the two components decide to collaborate by forming the link $\{i, j\}$, the Myerson fairness condition says that both players $i$ and $j$ gain equally from forming this link.

Since all members of a coalition are involved in the cooperation, one may argue that the fairness criterion should not be applied to the two individual players that are linked, but to the two components that are linked. This motivates the axiom of component fairness, which requires that if a link $\{i, j\}$ is deleted from $L$, the average loss in payoff in both components $K^{i}$ and $K^{j}$ is the same. Component fairness therefore emphasizes that in a cooperative graph game the gains associated to linking one component to the other should be attributed to the two components, rather 
than to the two individual players that form the link, and that the gains should be proportional to the size of the component.

Axiom 3.3 (Component fairness). A solution $f$ on the class of cycle-free graph games satisfies component fairness if, for any $(v, L)$, for any link $\{i, j\} \in L$, it holds that

$$
\frac{1}{\left|K^{i}\right|} \sum_{h \in K^{i}}\left(f_{h}(v, L)-f_{h}(v, L \backslash\{i, j\})\right)=\frac{1}{\left|K^{j}\right|} \sum_{h \in K^{j}}\left(f_{h}(v, L)-f_{h}(v, L \backslash\{i, j\})\right) .
$$

We now state the main theorem, which says that there is a unique solution that satisfies both axioms.

Theorem 3.4. On the class of cycle-free graph games, there is a unique solution that satisfies component efficiency and component fairness.

Proof. Suppose that $f$ satisfies component efficiency and component fairness. For any graph game $(v, L)$, let $l=|L|$ be the number of links and $c=\left|C_{m}^{L}(N)\right|$ the number of components in $(N, L)$. Observe that $l+c=n$, because the graph is cycle-free. Component efficiency implies that

$$
\sum_{h \in K} f_{h}(v, L)=v(K), \quad \text { for all } K \in C_{m}^{L}(N) .
$$

Also because of component efficiency we have $\sum_{h \in K^{i}} f_{h}(v, L \backslash\{i, j\})=v\left(K^{i}\right)$ and $\sum_{h \in K^{j}} f_{h}(v, L \backslash\{i, j\})=v\left(K^{j}\right)$ for any link $\{i, j\}$ in $L(K)$. Therefore, component fairness reduces to

$$
\frac{1}{\left|K^{i}\right|}\left(\sum_{h \in K^{i}} f_{h}(v, L)-v\left(K^{i}\right)\right)=\frac{1}{\left|K^{j}\right|}\left(\sum_{h \in K^{j}} f_{h}(v, L)-v\left(K^{j}\right)\right),
$$

for all $\{i, j\} \in L(K)$ and $K \in C_{m}^{L}(N)$. $^{2}$ Since there are $c$ equations of type (3.1) and $l$ equations of type (3.2) and all the $c+l=n$ equations are linearly independent, these equations uniquely determine $f(v, L)$.

Having proved that component efficiency and component fairness uniquely determine a solution on the class of cycle-free graph games, we are now going to give a closed form expression for this solution. To do so, we first discuss some general properties of directed graphs. A directed graph is a pair $(N, D)$, where $N$ is a set of nodes and $D$ is a collection of directed edges, i.e. $D \subseteq\{(i, j) \mid i, j \in N, i \neq j\}$. If $(i, j) \in D$, then the node $j$ is a successor of $i$ and $i$ is a predecessor of $j$. We say that $j \neq i$ is a subordinate of $i$ if there is a sequence of directed edges $\left(i_{h}, i_{h+1}\right) \in D, h=1, \ldots, k$, such that $i_{1}=i$ and $i_{k+1}=j$. We denote the set of subordinates of $i$ in $(N, D)$ by $S_{D}(i)$ and denote $S_{D}^{c}(i)=S_{D}(i) \cup\{i\}$. For $K \in 2^{N}$, the directed graph $(K, D(K))$ with $D(K)=\{(i, j) \in D \mid i, j \in K\}$ is called the directed subgraph of $(N, L)$ on $K$. A directed graph $(N, D)$ is a tree if there is one node in $N$, called the root or top-node, having no predecessors in $D$ and there is a unique sequence of directed edges in $(N, D)$ from this node to any other

\footnotetext{
2 Observe that, contrary to the proof of the uniqueness of the Myerson value, we do not need induction on the number of links. For the Myerson value one first has to determine the values $f_{h}(v, L \backslash\{i, j\})$ for $h=i, j$.
} 
node in $N$. Let $(N, \bar{D})$ be the undirected graph induced by $(N, D)$, i.e. $\bar{D}=\{\{i, j\} \mid(i, j) \in D\}$. A directed graph $(N, D)$ is a forest if for every component $K$ of $(N, \bar{D})$ the directed subgraph $(K, D(K))$ is a tree. A directed graph $(N, D)$ represents a hierarchy on $N$ when $(N, D)$ is a forest. Clearly, in a forest $(N, D)$ we have that $S_{D}^{c}(j) \subseteq S_{D}(i)$ when $j$ is a subordinate of $i$.

Finally, let $(N, L)$ be a cycle-free undirected graph and let $K$ belong to $C_{m}^{L}(N)$. Then every node $i \in K$ induces a unique tree $T(i)$ on $K$ in the following way. For any $j \in K \backslash\{i\}$, take the unique path in $(K, L(K))$ from $i$ to $j$, then change the undirected edges on this path to directed edges in such a way that the first node in any ordered pair is the node that comes first on the path from $i$ to $j$. We define $L_{T(i)}(j)=\{h \in K \mid(j, h) \in T(i)\}$ as the set of successors of $j$ in $T(i)$, so $L_{T(i)}(j) \subseteq S_{T(i)}(j)$.

Notice that the undirected cycle-free subgraph $(K, L(K))$ induces $k=|K|$ different trees, one tree for each of the $k$ different nodes of $K$. For $K \in C_{m}^{L}(N)$, we denote by $T_{L}^{K}$ the collection of all $|K|$ trees on $K$ induced by the undirected graph $(N, L)$.

Let $(v, L)$ be a cycle-free graph game. Consider a component $K$ of $(N, L)$ and $i \in K$. We now associate to player $j$ in $T(i)$ payoffs $t_{j}^{i}(v, L)$ given by

$$
t_{j}^{i}(v, L)=v\left(S_{T(i)}^{c}(j)\right)-\sum_{h \in L_{T(i)}(j)} v\left(S_{T(i)}^{c}(h)\right) .
$$

The payoff $t_{j}^{i}(v, L)$ to player $j \in K$ is equal to the worth of the coalition consisting of player $j$ and all his subordinates in $T(i)$ minus the sum of the worths of the coalitions consisting of any successor of player $j$ and all subordinates of this successor in $T(i)$.

We now define the Average Tree solution as the solution assigning to each cycle-free graph game $(v, L)$ the payoff vector in which player $j$ in a component $K \in C_{m}^{L}(N)$ receives the average over $i \in K$ of the payoffs $t_{j}^{i}(v, L)$.

Definition 3.5 (Average tree solution). On the class of cycle-free graph games, the average tree (AT) solution assigns to any $(v, L)$ the payoff vector $\operatorname{AT}(v, L)$ given by

$$
\operatorname{AT}_{j}(v, L)=\frac{1}{|K|} \sum_{i \in K} t_{j}^{i}(v, L),
$$

where $j$ is a player belonging to component $K \in C_{m}^{L}(N)$.

Notice that the average tree solution is a weighted average of $n$ vectors, each of which can be easily computed. The following example illustrates.

Example 3.6. Let the graph game $(N, v, L)$ be given by $N=\{1,2,3\}, v(\{1,2\})=1, v(\{2,3\})=$ $v(N)=2, v(S)=0$, otherwise, and $L=\{\{1,2\},\{2,3\}\}$. The graph $(N, L)$ is connected, so has a single component. The graph $(N, L)$ induces 3 trees, one for each of the 3 different nodes of $N$, $T(1)=\{(1,2),(2,3)\}, T(2)=\{(2,1),(2,3)\}$, and $T(3)=\{(3,2),(2,1)\}$. It follows immediately by Eq. (3.3) that $t^{1}(v, L)=(0,2,0)^{\top}, t^{2}(v, L)=(0,2,0)^{\top}$, and $t^{3}(v, L)=(0,1,1)^{\top}$, so

$$
\operatorname{AT}(v, L)=\frac{1}{3}\left(\begin{array}{l}
0 \\
2 \\
0
\end{array}\right)+\frac{1}{3}\left(\begin{array}{l}
0 \\
2 \\
0
\end{array}\right)+\frac{1}{3}\left(\begin{array}{l}
0 \\
1 \\
1
\end{array}\right)=\left(\begin{array}{c}
0 \\
5 / 3 \\
1 / 3
\end{array}\right)
$$


By the tree structure of $T(i)$ we have that for every player $j \in K$ the sets $S_{T(i)}^{c}(h), h \in$ $L_{T(i)}(j)$, form a partitioning of $S_{T(i)}(j)$ and thus

$$
t_{j}^{i}(v, L)=v\left(S_{T(i)}^{c}(j)\right)-v^{L}\left(S_{T(i)}(j)\right),
$$

where the last term is the restricted worth of the coalition consisting of all subordinates of $j$ in the tree $T(i)$. The payoff to player $j$ in tree $T(i)$ is therefore equal to what player $j$ contributes when he joins his subordinates in $T(i)$. Clearly, the set $S_{T(i)}^{c}(j)$ itself is connected, so when joining his subordinates, player $j$ connects all the subsets of subordinates of his successors to one connected set and receives his marginal contribution to it. Observe that a player $j \in K$ receives his own worth $v(\{j\})$ when $j$ has no subordinates in the tree $T(i)$. More generally, the total payoff to a player $j$ and all his subordinates in $T(i)$ is equal to the worth of the coalition $S_{T(i)}^{c}(j)$, i.e.

$$
\sum_{h \in S_{T(i)}^{c}(j)} t_{h}^{i}(v, L)=v\left(S_{T(i)}^{c}(j)\right), \quad j \in K .
$$

The next theorem shows that the AT-solution satisfies component efficiency and component fairness.

Theorem 3.7. The average tree solution satisfies component efficiency and component fairness.

Proof. Let $(v, L)$ be a graph game, and let $K$ be a component of $(N, v)$. Since player $i$ is the top-player in the tree $T(i)$ on $K$, and thus $S_{T(i)}^{c}(i)=K$, it follows from Eq. (3.5) that for any $i \in K$

$$
\sum_{h \in K} t_{h}^{i}(v, L)=\sum_{h \in S_{T(i)}^{c}(i)} t_{h}^{i}(v, L)=v\left(S_{T(i)}^{c}(i)\right)=v(K) .
$$

Hence,

$$
\sum_{h \in K} \operatorname{AT}_{h}(v, L)=\sum_{h \in K} \frac{1}{|K|} \sum_{i \in K} t_{h}^{i}(v, L)=\frac{1}{|K|} \sum_{i \in K} \sum_{h \in K} t_{h}^{i}(v, L)=\frac{1}{|K|} \sum_{i \in K} v(K)=v(K),
$$

which shows component efficiency.

To show component fairness, suppose link $\{i, j\}$ is deleted from component $K$. Component efficiency implies that for the components $K^{i}$ and $K^{j}$ in the graph $(N, L \backslash\{i, j\})$ it holds that $\sum_{h \in K^{i}} \mathrm{AT}_{h}(v, L \backslash\{i, j\})=v\left(K^{i}\right)$ and $\sum_{h \in K^{j}} \mathrm{AT}_{h}(v, L \backslash\{i, j\})=v\left(K^{j}\right)$.

Next, we compute $\operatorname{AT}(v, L)$. Consider again the component $K$ and the link $\{i, j\}$. For $h \in K^{i}$ we have by definition of $t^{h}(v, L)$ that

$$
\sum_{h^{\prime} \in K^{j}} t_{h^{\prime}}^{h}(v, L)=v\left(K^{j}\right)
$$

On the other hand, for $h \in K^{j}$ it holds that

$$
\sum_{h^{\prime} \in K^{j}} t_{h^{\prime}}^{h}(v, L)=v(K)-v\left(K^{i}\right),
$$

since by (3.6) $\sum_{h^{\prime} \in K^{i}} t_{h^{\prime}}^{h}(v, L)=v\left(K^{i}\right)$ and $\sum_{h^{\prime} \in K} t_{h^{\prime}}^{h}=v(K)$. There are $\left|K^{i}\right|$ equations of type (3.6) and $\left|K^{j}\right|$ equations of type (3.7). Therefore, it follows that

$$
\sum_{h \in K^{j}} \operatorname{AT}_{h}(v, L)=\frac{1}{|K|}\left(\left|K^{i}\right| v\left(K^{j}\right)+\left|K^{j}\right|\left(v(K)-v\left(K^{i}\right)\right)\right) .
$$


With $\left|K^{i}\right|+\left|K^{j}\right|=|K|$ it follows that

$$
\begin{aligned}
& \sum_{h \in K^{j}}\left(\operatorname{AT}_{h}(v, L)-\mathrm{AT}_{h}(v, L \backslash\{i, j\})\right) \\
& \quad=\frac{1}{|K|}\left(\left|K^{i}\right| v\left(K^{j}\right)+\left|K^{j}\right|\left(v(K)-v\left(K^{i}\right)\right)\right)-v\left(K^{j}\right) \\
& \quad=\frac{\left|K^{j}\right|}{|K|}\left(v(K)-v\left(K^{i}\right)-v\left(K^{j}\right)\right) .
\end{aligned}
$$

Analogously, it follows that

$$
\sum_{h \in K^{i}}\left(\mathrm{AT}_{h}(v, L)-\mathrm{AT}_{h}(v, L \backslash\{i, j\})\right)=\frac{\left|K^{i}\right|}{|K|}\left(v(K)-v\left(K^{i}\right)-v\left(K^{j}\right)\right),
$$

which shows that AT satisfies component fairness.

The next corollary follows immediately from Theorems 3.4 and 3.7.

Corollary 3.8. On the class of cycle-free graph games, the average tree solution is the unique solution that satisfies component efficiency and component fairness.

\section{Stability}

In this section we show that within the class of superadditive cycle-free graph games the average tree solution can also be characterized as the unique solution that satisfies component fairness and that belongs to the core. Consider a connected cycle-free graph $(N, L)$, so that $N$ is the unique component. Then the AT-solution becomes

$$
\operatorname{AT}_{j}(v, L)=\frac{1}{n} \sum_{i \in N} t_{j}^{i}(v, L), \quad j \in N .
$$

Suppose that $v$ is superadditive. It has been shown by Demange (2004) that the vector $t^{i}(v, L)$ in which a player $j$ gets a payoff equal to the worth of the coalition consisting of himself with all his subordinates minus the restricted worth of the coalition of his subordinates is in the core of the restricted game $v^{L}$. In fact, it holds that $v^{L}$ is permutationally convex for any permutation $\pi$ satisfying $\pi_{j}<\pi_{h}$ when $j$ is a subordinate of $h$ on a tree $T(i)$ on $N$, see for instance van Velzen (2005). According to Granot and Huberman (1982) this implies that the corresponding marginal vector $m^{\pi}$ is in the core of $v^{L}$. It is straightforward to verify that $t^{i}(v, L)=m^{\pi}\left(v^{L}\right)$ for any permutation $\pi$ that satisfies the condition above for the tree $T(i)$. Since the core is convex and $\operatorname{AT}(v, L)$ is the average of all vectors $t^{i}(v, L), i \in N$, this implies that the AT-solution is in the core of $v^{L}$ and thus cannot be dominated by any coalition. The discussion above generalizes straightforwardly to the case where the graph $(N, L)$ consists of several components. Since stability, the property that the solution is in the core, implies the weaker condition of component efficiency, we have the following theorem.

Theorem 4.1. On the class of cycle-free superadditive graph games the AT-solution is the unique solution satisfying stability and component fairness. 
The theorem not only says that the AT-solution is the unique stable solution satisfying component fairness, it also says that there exists a solution being stable and component fair. This makes the AT-solution an attractive alternative to other solutions such as the Myerson value and the position value which may not be stable, even not on the class of superadditive cycle-free graph games.

The Myerson value $\mu(v, L)$, characterized by component efficiency and fairness, satisfies, for any link $\{i, j\} \in L$,

$$
\mu_{i}(v, L)-\mu_{i}(v, L \backslash\{i, j\})=\mu_{j}(v, L)-\mu_{j}(v, L \backslash\{i, j\}) .
$$

It is equal to the Shapley value $\psi\left(v^{L}\right)$ of the restricted game $v^{L}$, implying that it may be outside the core of $v^{L}$ when $v^{L}$ is not convex. Fairness may contradict the requirement of stability, whereas component fairness does not. Also the position value, denoted by $\gamma(v, L)$, introduced for the class of all graph games and characterized on the class of cycle-free graph games in Borm et al. (1992), does not need to be stable. Slikker (2005) characterizes the position value on the class of graph games as the unique solution satisfying component efficiency and balanced total threats. The total threat of a player $i$ towards another player $j$ is defined as the sum over all links of player $i$ of the payoff differences player $j$ experiences if such a link is broken, i.e. for each pair of players $i, j \in N$ it holds that

$$
\sum_{h \mid\{i, h\} \in L}\left(\gamma_{j}(v, L)-\gamma_{j}(v, L \backslash\{i, h\})\right)=\sum_{h \mid\{j, h\} \in L}\left(\gamma_{i}(v, L)-\gamma_{i}(v, L \backslash\{j, h\})\right) .
$$

Balanced total threats means that the total threat of any player $i$ towards another player $j$ is equal to the total threat of player $j$ towards player $i$.

The next example shows that also the balanced threat property may contradict the requirement of stability, i.e. also the position value may be outside the core.

Example 4.2. As in Example 3.6, let the graph game $(N, v, L)$ be given by $N=\{1,2,3\}$, $v(\{1,2\})=1, v(\{2,3\})=v(N)=2, v(S)=0$, otherwise, and $L=\{\{1,2\},\{2,3\}\}$. Observe that $v^{L}=v$, so that the Myerson value equals the Shapley value: $\mu(v, L)=\psi\left(v^{L}\right)=\psi(v)=$ $(1 / 6,7 / 6,2 / 3)^{\top}$. To obtain the position value, straightforward calculations show that $\gamma(v, L \backslash$ $\{1,2\})=(0,1,1)^{\top}$ and $\gamma(v, L \backslash\{2,3\})=\left(\frac{1}{2}, \frac{1}{2}, 0\right)^{\top}$. Then, it follows that $\gamma(v, L)=\left(\frac{1}{4}, 1, \frac{3}{4}\right)^{\top}$ by solving the system of three equations given by the component efficiency property and the two conditions implied by balanced threats between 1 and 2 and between 2 and 3. Since $x_{1}=0$ for any payoff vector $x$ in the core of $v^{L}$, this shows that both the Myerson value and the position value are not stable. Finally, Example 3.6 computed AT $(v, L)$ to be equal to $(0,5 / 3,1 / 3)$, a vector that belongs to the core.

\section{Harsanyi dividends}

In this section, we compare the three values in terms of distributions of the so-called Harsanyi dividends. Let $\Omega$ be the collection of non-empty subsets of $N$. Then, for $T \in \Omega$, the unanimity game $u_{T}$ on $N$ is given by $u_{T}(S)=1$ if $T \subseteq S$, and $u_{T}(S)=0$ otherwise. Leaving out $v(\emptyset)$, it is well known that the collection of unanimity games forms a basis in $\mathbb{R}^{2^{n}-1}$ for the class of TU-games on $N$, i.e. for any game $v$ represented as a $\left(2^{n}-1\right)$-vector with the worths of the non-empty coalitions as its components, it holds that

$$
v=\sum_{S \in \Omega} \Delta^{S}(v) u_{S}
$$


where the coefficients $\Delta^{S}(v)$ are the Harsanyi dividends, see Harsanyi (1959), given by

$$
\Delta^{S}(v)=\sum_{T \subseteq S}(-1)^{|S|-|T|} v(T), \quad S \in \Omega .
$$

It is well known that the Shapley value can be obtained by distributing the Harsanyi dividend of coalition $S$ equally among the players in $S$,

$$
\psi_{i}(v)=\sum_{S \in \Omega \mid i \in S} \frac{1}{|S|} \Delta^{S}(v), \quad i \in N
$$

The Shapley payoff to player $i$ is equal to the sum over all coalitions $S$ containing player $i$ of the dividend of coalition $S$ divided by $|S|$.

Consider a cycle-free graph game $(v, L)$. For a connected set $S$, a node $i \in S$ is extreme if $S \backslash\{i\}$ is connected in the subgraph $(N \backslash\{i\}, L(N \backslash\{i\}))$. The set of extreme nodes of $S$ is denoted by $\operatorname{Ex}(S)$. Owen (1986), see also Borm et al. (1992), showed that the dividends of the restricted game can be expressed as sum of the dividends of the underlying game $v$,

$$
\Delta^{S}\left(v^{L}\right)= \begin{cases}\sum_{T \in \Sigma(S)} \Delta^{T}(v), & \text { if } S \text { is connected } \\ 0, & \text { otherwise }\end{cases}
$$

where $\Sigma(S)=\{T \subseteq S \mid \operatorname{Ex}(S) \subseteq T\}$. Since the dividends of not connected coalitions are zero, for the restricted game of a cycle-free graph game $(v, L)$ Eq. $(5.8)$ reduces to

$$
v^{L}=\sum_{S \in C^{L}(N)} \Delta^{S}\left(v^{L}\right) u_{S}
$$

and the payoffs according to the Myerson value follow by distributing the dividends of the connected coalitions equally among its players

$$
\mu_{i}(v, L)=\sum_{S \in C^{L}(N) \mid i \in S} \frac{1}{|S|} \Delta^{S}\left(v^{L}\right), \quad i \in N .
$$

As follows from Borm et al. (1992), see also van den Brink et al. (2004), the position value is obtained by distributing the dividend of any connected coalition $S$ proportional among its players according to the degrees of the players in the subgraph $(S, L(S))$, i.e. the payoff of player $i$ according to the position value is given by

$$
\gamma_{i}(v, L)=\sum_{S \in C^{L}(N) \mid i \in S} \frac{d_{S}^{L}(i)}{\sum_{j \in S} d_{S}^{L}(j)} \Delta^{S}\left(v^{L}\right), \quad i \in N .
$$

Comparing the two values shows that in the position value the more central players, that means players with more neighbors, get higher shares in the dividends. ${ }^{3}$

Also the average tree solution can be obtained by distributing the dividends appropriately. To derive these distributions, for each connected $S$ in $(N, L)$ and $j \in S$, let $p_{S}^{L}(j)$ be the number of players outside $S$ represented by $j$ in $S$. We say that player $j \in S$ represents player $k$ outside $S$,

\footnotetext{
3 Since dividends may be negative, this does not necessarily mean that in the position value the more central players get higher payoffs than in the Myerson value. In the example above, the coalition $\{1,2\}$ has dividend 1 , coalition $\{2,3\}$ has dividend 2 and the grand coalition $N$ has dividend -1 . Since in the latter coalition player 2 has degree two and the other players degree one, the position value gives a lower payoff to player 2 than the Myerson value.
} 
if $k$ is connected to $j$ and on the unique path connecting $j$ and $k$ all players between $j$ and $k$ are outside $S$. So,

$$
p_{S}^{L}(j)=\sum_{h \in N \backslash S \mid\{j, h\} \in L}\left|K^{h}\right|,
$$

where, as in the previous section, $K^{h}$ is the component of the graph $(N, L \backslash\{j, h\})$ containing player $h$. Clearly, player $j$ connects the players in $S$ with the players in $K^{h}$ in the sense that link $\{j, h\}$ is on the path between any player $i$ in $S$ and any player $k$ in the set $K^{h} \subseteq N \backslash S$. Notice that for some players $j, p_{S}^{L}(j)=0$. We now have the following result.

Theorem 5.1. The payoff of player $i$ according to the AT-solution is given by

$$
A T_{i}(v, L)=\sum_{S \in C^{L}(N) \mid i \in S} \frac{1+p_{S}^{L}(i)}{|S|+\sum_{j \in S} p_{S}^{L}(j)} \Delta^{S}\left(v^{L}\right), \quad i \in N
$$

Proof. First, observe that AT is linear, i.e. for two games $v$ and $w$ and coefficients $\alpha, \beta \in \mathbb{R}$, it holds that $\mathrm{AT}(\alpha v+\beta w, L)=\alpha \operatorname{AT}(v, L)+\beta \mathrm{AT}(w, L)$. This follows from the fact that the AT-solution is the average of specific marginal vectors of the restricted game $v^{L}$, where the choice of the marginal vectors is independent of the payoffs. Further, observe that $u_{S}^{L}=u_{S}$ if $S$ is connected in $(N, L)$. Hence, with Eq. (5.10) it follows that

$$
\operatorname{AT}_{i}(v, L)=\sum_{S \in C^{L}(N)} \operatorname{AT}_{i}\left(u_{S}, L\right) \Delta^{S}\left(v^{L}\right), \quad i \in N
$$

Let $S$ be a connected coalition in $(N, L)$ and let $T \in C_{m}^{L}(N)$ be the component of $(N, L)$ containing $S$. Since $u_{S}(K)=0$ for each component $K \neq T$, it follows from component efficiency and component fairness that $\operatorname{AT}_{j}\left(u_{S}, L\right)=0$ for all $j \notin T$. Further $u_{S}(T)=1$, so component efficiency requires that $\sum_{j \in T} \operatorname{AT}_{j}\left(u_{S}, L\right)=1$.

Consider any link $\{i, j\}$ involving at least one player outside $S$. The graph $L \backslash\{i, j\}$ has two components being a subset of $T$, say $K^{i}$ and $K^{j}$, where $i \in K^{i}$ and $j \in K^{j}$. Moreover, without loss of generality, $S \subseteq K^{j}$. Since $u_{S}\left(K^{i}\right)=0$ and $u_{S}\left(K^{j}\right)=1$, component efficiency requires that $\sum_{h \in K^{i}} \mathrm{AT}_{h}\left(u_{S}, L \backslash\{i, j\}\right)=0$ and $\sum_{h \in K^{j}} \mathrm{AT}_{h}\left(u_{S}, L \backslash\{i, j\}\right)=1$. Component fairness implies that $\sum_{h \in K^{i}} \mathrm{AT}_{h}\left(u_{S}, L\right)=0$ and $\sum_{h \in K^{j}} \mathrm{AT}_{h}\left(u_{S}, L\right)=1$. Since this holds for any link $\{i, j\}$ involving at least one player outside $S$, it follows that $\operatorname{AT}_{i}\left(u_{S}, L\right)=0$ for all $i \in T \backslash S$ and thus for all $i$ not in $S$. Consequently, $\sum_{j \in S} \operatorname{AT}_{j}\left(u_{S}, L\right)=1$.

Now, for any two linked players $i$ and $j$ in $S$, let $u_{S}^{L \backslash\{i, j\}}$ be the restricted game of $u_{S}$ on $(N, L \backslash\{i, j\})$. Since $S$ is not connected anymore, $u_{S}^{L \backslash\{i, j\}}(T)=0$ for any $T \in 2^{N}$, i.e. the restricted game is the null-game, yielding worth zero to any coalition. Consequently, $\mathrm{AT}_{j}\left(u_{S}, L \backslash\{i, j\}\right)=0$ for all $j$. Hence the component fairness property requires that for each pair $i, j \in S$ with $\{i, j\} \in L$,

$$
\frac{1}{\left|K^{i}\right|} \sum_{h \in K^{i}} \operatorname{AT}_{h}\left(u_{S}, L\right)=\frac{1}{\left|K^{j}\right|} \sum_{h \in K^{j}} \operatorname{AT}_{h}\left(u_{S}, L\right) .
$$


Since $S$ is connected, there are $|S|-1$ links in $S$ and thus $|S|-1$ of such equations. Together with $\sum_{i \in S} \operatorname{AT}_{i}\left(u_{S}, L\right)=1$ we have a system of $|S|$ linearly independent equations with $|S|$ unknowns, yielding a unique solution. It is easy to verify that the solution is given by

$$
\operatorname{AT}_{i}\left(u_{S}, L\right)=\frac{1+p_{S}^{L}(i)}{|S|+\sum_{j \in S} p_{S}^{L}(j)}, \quad i \in S .
$$

Doing this for any connected $S$, the theorem follows from Eq. (5.10) and the linearity of the AT-solution.

Theorem 5.1 shows that the AT-solution can be obtained by distributing the dividend of any connected coalition $S$ among the players in $S$ in such a way that the share of player $i \in S$ in the dividend of coalition $S$ is given by $\left(1+p_{S}^{L}(i)\right) /\left(|S|+\sum_{j \in S} p_{S}^{L}(j)\right)$. The weight $1+p_{S}^{L}(i)$ is equal to the number of players on behalf of which player $i$ acts in $S$, including himself, i.e. one plus the number of players outside $S$ in the component containing $S$ and being connected to $i$ through a path of players outside $S$. Recall that the Myerson value is obtained by distributing the dividend of any connected coalition equally among the players in $S$ and the position value is obtained by distributing the dividends according to the degree of each player in the subgraph $(S, L(S))$. In both cases the shares of the players in the dividends only depend on the structure of the subgraph $(S, L(S))$. In fact, the Myerson value only depends on the number of players in $S$. In contrast, for the AT-solution the shares also depend on the structure of the graph $(N, L)$ outside $S$. Indeed, the shares depend on the structure of the whole subgraph on the component $K$ containing $S$. As a result, the AT-solution for the unanimity game $u_{S}$ may change when the graph changes on $K$ but not on $S$, whereas the other two solutions can only change when the graph changes on $S$ itself. More precisely, when $\{i, j\}$ is a link of $L$ not on $S$ but on the component $K$ containing $S$, we have that

$$
\mu\left(u_{S}, L\right)=\mu\left(u_{S}, L \backslash\{i, j\}\right) \quad \text { and } \quad \gamma\left(u_{S}, L\right)=\gamma\left(u_{S}, L \backslash\{i, j\}\right),
$$

but

$$
\mathrm{AT}\left(u_{S}, L\right) \neq \mathrm{AT}\left(u_{S}, L \backslash\{i, j\}\right) .
$$

As has been shown in Borm et al. (1992), both the Myerson value and the position value satisfy the so-called superfluous link property. A link $\{i, j\} \in L$ is superfluous in a graph game $(v, L)$ if $v^{E}(N)=v^{E \cup\{i, j\}}(N)$ for all $E \subseteq L \backslash\{i, j\}$, so when for each subset of links $E$ not containing $\{i, j\}$, the restricted value of the grand coalition on the graph $(N, E)$ is equal to the restricted value of it on the graph $(N, E \cup\{i, j\})$. A solution $f$ satisfies the superfluous link property if $f(v, L)=f(v, L \backslash\{i, j\})$ whenever $\{i, j\}$ is a superfluous link in $(v, L)$. Clearly, any link outside a connected coalition $S$ is a superfluous link in the graph game $\left(u_{S}, L\right)$. Since deleting such a link may change the AT-solution, AT does not satisfy the superfluous link property.

Theorem 5.1 is very useful when calculating the average tree solution. The following example illustrates this and also highlights once more the core property of the average tree solution.

Example 5.2. Consider the graph game $(v, L)$, with $v(N)=10, v(\{2, \ldots, n\})=10, v(S)=8$ if $\{1,2\} \subset S \neq N$, and $v(S)=0$, otherwise, where $n \geqslant 3$. The communication graph is given by the line graph $L=\{\{i, i+1\} \mid i \in N \backslash\{n\}\}$.

The crux of the example is that players 1 and 2 can obtain a payoff of 8 by themselves, and a payoff of 10 if all players collaborate. On the other hand, player 2 together with all other players but player 1, can also obtain 10. Therefore, in any core solution, player 1 obtains 0 and player 2 
receives at least 8 . Together with efficiency (the sum of the payoffs is 10) and non-negativity of the payoffs, these conditions characterize the core.

The Harsanyi dividends are as follows

$$
\Delta^{\{1,2\}}=8, \quad \Delta^{\{2, \ldots, n\}}=10, \quad \Delta^{N}=-8 .
$$

To obtain the Myerson value, the Harsanyi dividends are divided equally among the participating players. So, $\Delta^{\{1,2\}}$ leads to payoffs 4 for both players 1 and $2, \Delta^{\{2, \ldots, n\}}$ gives rise to $10 /(n-1)$ for players $2, \ldots, n$, and $\Delta^{N}$ to $-8 / n$ for all players. We find that $\mu_{1}(v, L)=4-8 / n$, $\mu_{2}(v, L)=4+10 /(n-1)-8 / n$, and $\mu_{i}(v, L)=8 / n-10 /(n-1), i=3, \ldots, n$. When $n \rightarrow \infty$, $\mu_{1}(v, L) \rightarrow 4, \mu_{2}(v, L) \rightarrow 4$, and $\sum_{i=3}^{n} \mu_{i}(v, L) \rightarrow 2$. So, in the limit, the Myerson value allocates 4 to both players 1 and 2, whereas player 1 gets 0 in any core allocation, and player 2 at least 8 . For any $n \geqslant 3$, the Myerson value is not in the core.

For the position value the Harsanyi dividends are divided proportional to the degree of the participating players. So, $\Delta^{\{1,2\}}$ leads to payoffs 4 for both players 1 and $2, \Delta^{\{2, \ldots, n\}}$ gives rise to $5 /(n-2)$ for both players 2 and $n$ and $10 /(n-1)$ for players $3, \ldots, n-1$, and $\Delta^{N}$ leads to $-4 /(n-1)$ for both players 1 and $n$ and $-8 /(n-1)$ for players $2, \ldots, n-1$. We find that $\gamma_{1}(v, L)=4-4 /(n-1), \gamma_{2}(v, L)=4+5 /(n-2)-8 /(n-1), \gamma_{i}(v, L)=10 /(n-2)-8 /(n-$ $1), i=3, \ldots, n-1$, and $\gamma_{n}(v, L)=5 /(n-2)-4 /(n-1)$. Again, when $n \rightarrow \infty, \gamma_{1}(v, L) \rightarrow$ $4, \gamma_{2}(v, L) \rightarrow 4$, and $\sum_{i=3}^{n} \gamma_{i}(v, L) \rightarrow 2$. The position value leads to qualitatively the same allocation as the Myerson value, and does not belong to the core either for $n \geqslant 3$.

Finally, we turn to the average tree solution. By Theorem 5.1, the Harsanyi dividends $\Delta^{\{1,2\}}$ lead to payoffs $8 / n$ for player 1 and $8-8 / n$ for player $2, \Delta^{\{2, \ldots, n\}}$ gives rise to $20 / n$ for player 2 and $10 / n$ for players $3, \ldots, n$, and $\Delta^{N}$ to $-8 / n$ for all players. We find that $\operatorname{AT}_{1}(v, L)=0$, $\operatorname{AT}_{2}(v, L)=8+4 / n$, and $\operatorname{AT}_{i}(v, L)=2 / n, i=3, \ldots, n$. When $n \rightarrow \infty, \operatorname{AT}_{1}(v, L) \rightarrow 0$, $\operatorname{AT}_{2}(v, L) \rightarrow 8$, and $\sum_{i=3}^{n} \operatorname{AT}_{i}(v, L) \rightarrow 2$. For $n$ large, the average tree solution allocates 0 to player 1 and 8 to player 2 . The average tree solution belongs to the core for all values of $n$, as was demonstrated in general in Theorem 4.1.

The example is also useful to highlight the difference between fairness and component fairness. Consider the game $(v, L \backslash\{1,2\})$. For the Myerson value, we find that $\mu_{1}(v, L \backslash\{1,2\})=0$ and $\mu_{i}(v, L \backslash\{1,2\})=10 /(n-1), i=2, \ldots, n$. As predicted by fairness, the increase in payoff for players 1 and 2 is the same when link $\{1,2\}$ is added, and happens to be equal to $4-8 / n$. Using Theorem 5.1 once more, we compute that $\operatorname{AT}(v, L \backslash\{1,2\})=\mu(v, L \backslash\{1,2\})$. As predicted by component fairness, the increase in payoff for player 1 is equal to the average increase in payoff for players $2, \ldots, n$, and can be verified to be equal to 0 .

\section{Concluding remarks}

In this paper we propose a new solution for cycle-free graph games. In such games players are only able to cooperate if they are connected to each other. In case of superadditivity, the core of the game is non-empty and contains for each player a specific marginal vector. This marginal vector is induced by a tree in which that player is the root and the directed edges correspond to the undirected edges of the graph. The solution is then the average of these marginal vectors. Since all these marginal vectors are an element of the core, the new solution is also an element of the core, even if the game is not convex. This is in contrast to the Myerson value and the position value. These values may be outside the core.

The new solution has been axiomatized by component efficiency and component fairness. The latter property says that if a link is deleted, the average loss per player is the same in the 
two subcomponents resulting after deletion of the link. This means that the surplus in worth obtained from a link is distributed over these two subcomponents proportional to their number of players. In this respect the new solution differs from the Myerson value and the position value. The Myerson value is characterized by component efficiency and by fairness, the property that if a link is deleted, then the two players that were connected by this link have equal loss. This loss is therefore not related to the size of the components resulting after the deletion of the link. The position value is characterized by component efficiency and balanced total threats, the property that the total threat of a player towards another player is equal to the total threat of the latter player towards the former player. The average tree solution may fail to satisfy balanced total threats.

In terms of distributions of Harsanyi dividends the new solution is obtained by distributing the dividends of a connected coalition among its members in such a way that the share of a player in it is given by the relative number of players on which behalf the player acts in the coalition, including himself. This means that the shares depend on the structure of the subgraph on the component of which the coalition is a subset and not just on the structure of the subgraph on the coalition itself as is the case for the Myerson value and the position value. This property guarantees that the value is an element of the core.

It is not hard to compute the average tree solution. If $n$ is the number of players and the graph has one component, the value can be calculated as follows. First, for each player $i$, a permutation $\pi^{i}$ is determined satisfying that player $j$ has a higher rank than player $k$ if player $j$ lies on the unique path connecting player $i$ and player $k$. Any permutation that satisfies this condition leads to the same marginal vector. Next, the average tree solution is the average of the $n$ marginal vectors obtained in this way. The calculation is analogous for the case the graph has more than one component.

\section{Acknowledgment}

The first author would like to thank the Netherlands organization for Scientific Research (NWO) for financial support.

\section{References}

Bondareva, O.N., 1963. Some applications of linear programming methods to the theory of cooperative games. Problemy Kibernet. 10, 119-139.

Borm, P., Owen, G., Tijs, S., 1992. On the position value for communication situations. SIAM J. Discrete Math. 5, 305-320.

van den Brink, R., van der Laan, G., Pruzhansky, V., 2004. Harsanyi power solutions for graph-restricted games. TI discussion paper 2004-095/1, Tinbergen Institute, Amsterdam/Rotterdam.

Demange, G., 1994. Intermediate preferences and stable coalition structures. J. Math. Econ. 23, 45-58.

Demange, G., 2004. On group stability in hierarchies and networks. J. Polit. Economy 112, 754-778.

Gillies, D.B., 1953. Some Theorems on $n$-Person Games. Princeton Univ. Press, Princeton.

Granot, G., Huberman, G., 1982. The relationship between convex games and minimal cost spanning tree games: A case for permutationally convex games. SIAM J. Algebraic Discrete Methods 3, 288-292.

Harsanyi, J.C., 1959. A bargaining model for cooperative $n$-person games. In: Tucker, A.W., Luce, R.D. (Eds.), Contributions to the Theory of Games IV. Princeton Univ. Press, Princeton, pp. 325-355.

Kaneko, M., Wooders, M.H., 1982. Cores of partitioning games. Math. Soc. Sci. 3, 313-327.

Le Breton, M., Owen, G., Weber, S., 1992. Strongly balanced cooperative games. Int. J. Game Theory 20, $419-427$.

Meessen, R., 1988. Communication games. Master thesis, University of Nijmegen.

Myerson, R.B., 1977. Graphs and cooperation in games. Math. Oper. Res. 2, 225-229.

Myerson, R.B., 1980. Conference structures and fair allocation rules. Int. J. Game Theory 9, 169-182. 
Owen, G., 1986. Values of graph-restricted games. SIAM J. Algebraic Discrete Methods 7, 210-220.

Shapley, L., 1953. A value for $n$-person games. In: Kuhn, H.W., Tucker, A.W. (Eds.), Contributions to the Theory of Games II. Princeton Univ. Press, Princeton, pp. 307-317.

Slikker, M., 2005. A characterization of the position value. Int. J. Game Theory 33, 505-514.

van Velzen, S., 2005. Cooperation in network and scheduling. PhD thesis, CentER, Tilburg University. 